Notfall Rettungsmed $2015 \cdot 18: 173$

DOI 10.1007/s10049-015-0024-x

○) Springer-Verlag Berlin Heidelberg 2015

\section{B.W. Böttiger ${ }^{1}$ C. Waydhas ${ }^{2} \cdot$ C. Wrede ${ }^{3}$}

${ }^{1}$ Klinik für Anästhesiologie und Operative Intensivmedizin, Universitätsklinikum Köln, Köln, Deutschland

2 Klinik für Unfallchirurgie, Universitätsklinikum Essen, Essen, Deutschland

${ }^{3}$ Interdisziplinäres Notfallzentrum HELIOS Klinikum Berlin-Buch, Berlin, Deutschland

\title{
Ein Originalienheft der Notfall+Rettungsmedizin - interdisziplinär und interprofessionell
}

Liebe Leserinnen und Leser,

diese Ausgabe der Notfall+Rettungsmedi$z i n$ ist ausnahmsweise keinem Leitthema gewidmet. Stattdessen finden Sie eine $\mathrm{Zu}$ sammenstellung ganz unterschiedlicher Originalien und einer Kasuistik, welche auch die Interdisziplinarität und die Interprofessionalität unserer Zeitschrift widerspiegelt. Diese Beiträge wurden in den letzten Monaten frei bei der Notfall+Rettungsmedizin eingereicht und von Herausgebern und Schriftleitung zur Publikation angenommen. Wir danken allen Autoren für ihren wichtigen Beitrag zu unserer Zeitschrift.

Ebenso finden Sie in dieser Ausgabe das Positionspapier für eine Reform der medizinischen Notfallversorgung in deutschen Notaufnahmen, welches mit Unterstützung der Deutschen Interdisziplinären Vereinigung für Intensiv- und Notfallmedizin (DIVI), der Deutschen Gesellschaft für Internistische Intensiv- und Notfallmedizin (DGIIN), der Deutschen Gesellschaft für Anästhesiologie und Intensivmedizin (DGAI), der Deutschen Gesellschaft für Chirurgie (DGCH) und der Deutschen Gesellschaft Interdisziplinäre Notfall- und Akutmedizin (DGINA) entstanden ist. Dieses möchten wir als einen Diskussionsbeitrag zur Lage der Notfallversorgung in deutschen Notaufnahmen verstehen. Wir möchten Ihnen diesen Beitrag unbedingt präsentieren, da wir es als Schriftleiter einer notfall- und rettungsmedizinischen Zeitschrift für unsere Aufgabe halten, ein breites und manchmal auch kontroverses Themenspektrum anzubieten. Gerne möchten wir eine Diskussion mit Ihnen darüber beginnen und würden uns über zahlreiche Zusendungen freuen. Kommentieren Sie den Beitrag online auf springermedizin.de, geben Sie dazu den Beitragstitel in die Suche ein oder gehen Sie über den unten stehenden QR-Code und nutzen Sie anschließend die Kommentarfunktion am Beitragsende oder schicken Sie einen Leserbrief für die Publikation in der Zeitschrift an die Verlagsredaktion (ines.wolff@springer.com).

Wir wünschen Ihnen eine gewinnbringende Lektüre aller Beiträge.

Prof. Dr. B.W. Böttiger

Prof. Dr. C. Waydhas

Prof. Dr. C. Wrede 